\title{
ERRATUM
}

\section{Ultrathin conformal devices for precise and continuous thermal characterization of human skin}

R. Chad Webb, Andrew P. Bonifas, Alex Behnaz, Yihui Zhang, Ki Jun Yu, Huanyu Cheng, Mingxing Shi, Zuguang Bian, Zhuangjian Liu, Yun-Soung Kim, Woon-Hong Yeo, Jae Suk Park, Jizhou Song, Yuhang Li, Yonggang Huang, Alexander M. Gorbach and John A. Rogers

Nature Materials 12, 938-944 (2013); published online 15 September 2013; corrected after print 26 September 2013.

In the version of this Article originally published, in Fig. 3b,c the labels at the top of the graphs were missing. This error has been corrected in the HTML and PDF versions of the Article. 